\title{
The upGREAT Dual Frequency Heterodyne Arrays for SOFIA
}

\author{
C. Risacher ${ }^{1,6,9}$, R. Güsten ${ }^{1}$, J. Stutzki ${ }^{2}$, H.-W. Hübers ${ }^{3}$, R. Aladro ${ }^{1}$, A. Bell ${ }^{1}$, C. Buchbender ${ }^{2}$, \\ D. Büchel ${ }^{2}$, T. Csengeri ${ }^{1}$, C. Duran ${ }^{1}$, U. U. Graf ${ }^{2}$, R. D. Higgins ${ }^{2}$, C. E. Honingh ${ }^{2}$, K. Jacobs ${ }^{2}$,

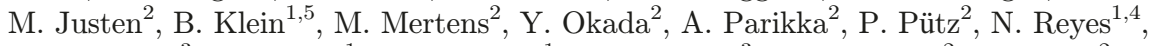 \\ H. Richter ${ }^{3}$, O. Ricken ${ }^{1}$, D. Riquelme ${ }^{1}$, N. Rothbart ${ }^{3}$, N. Schneider ${ }^{2}$, R. Simon ${ }^{2}$,

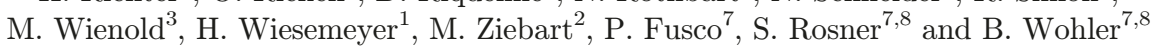 \\ ${ }^{1}$ Max-Planck-Institut für Radioastronomie, Auf dem Hügel 69, 53121, Bonn, Germany \\ ${ }^{2}$ I. Physikalisches Institut der Universität zu Köln \\ Zülpicher Strasse 77, 50937 Köln, Germany \\ ${ }^{3}$ Institute of Optical Sensor Systems, German Aerospace Center (DLR) \\ Rutherfordstr. 2, 12489 Berlin, Germany \\ ${ }^{4}$ Departamento de Ingeniería Eléctrica, Universidad de Chile, Santiago, Chile \\ ${ }^{5}$ University of Applied Sciences Bonn-Rhein-Sieg \\ Sankt Augustin, 53757 Germany \\ ${ }^{6}$ IRAM, 300 rue de la Piscine, 38406 Saint Martin d'Heres, France \\ ${ }^{7}$ NASA Ames Research Center, Moffett Field, CA 94035, USA \\ ${ }^{8}$ SETI Institute, Mountain View, CA 94043, USA \\ ${ }^{9}$ risacher@iram.fr
}

Received September 14, 2018; Accepted November 4, 2018; Published December 3, 2018

\begin{abstract}
We present the performance of the upGREAT heterodyne array receivers on the SOFIA telescope after several years of operations. This instrument is a multi-pixel high resolution $\left(\mathrm{R} \gtrsim 10^{7}\right)$ spectrometer for the Stratospheric Observatory for Far-Infrared Astronomy (SOFIA). The receivers use 7-pixel subarrays configured in a hexagonal layout around a central pixel. The low frequency array receiver (LFA) has $2 \times 7$ pixels (dual polarization), and presently covers the $1.83-2.07 \mathrm{THz}$ frequency range, which allows to observe the [CII] and [OI] lines at $158 \mu \mathrm{m}$ and $145 \mu \mathrm{m}$ wavelengths. The high frequency array (HFA) covers the [OI] line at $63 \mu \mathrm{m}$ and is equipped with one polarization at the moment ( 7 pixels, which can be upgraded in the near future with a second polarization array). The $4.7 \mathrm{THz}$ array has successfully flown using two separate quantum-cascade laser local oscillators from two different groups. NASA completed the development, integration and testing of a dual-channel closed-cycle cryocooler system, with two independently operable He compressors, aboard SOFIA in early 2017 and since then, both arrays can be operated in parallel using a frequency separating dichroic mirror. This configuration is now the prime GREAT configuration and has been added to SOFIA's instrument suite since observing cycle 6 .
\end{abstract}

Keywords: SOFIA (GREAT), high resolution spectroscopy, THz astronomy, airborne.

\section{Introduction - Scientific Motivation}

Since the turn-off of the HIFI/Herschel instrument (de Graauw et al., 2010; Pilbratt et al., 2010), there has been very limited number of high-resolution spectroscopy instruments capable of performing farinfrared wavelengths observations. For example, the balloon-borne Stratospheric Terahertz Observatory (STO-2) performed high-resolution observations in

\footnotetext{
${ }^{9}$ Corresponding author.

This is an Open Access article published by World Scientific Publishing Company. It is distributed under the terms of the Creative Commons Attribution 4.0 (CC-BY) License. Further distribution of this work is permitted, provided the original work is properly cited.
} 
the $\mathrm{THz}$ range in December 2016 for about $\sim 500 \mathrm{~h}$. On the other hand, the spectrometer GREAT (Heyminck et al., 2012) aboard the SOFIA airborne observatory (Young et al., 2012) is the only facility that provides regular high-resolution spectroscopy at far-infrared wavelengths.

GREAT saw first light on April 1st 2011 during SOFIA's early science phase, and since then has been operated on more than 135 science flights, collecting unique science data for more than $1000 \mathrm{~h}$. Starting as a dual-color single-pixel receiver operating in parallel in the 1.5 and $1.9 \mathrm{THz}$ frequency bands, a major upgrade occurred in 2015 when the low-frequency array receiver upGREAT/LFA was installed, spatially multiplexing now with $2 \times 7$ pixels in the $1.9 \mathrm{THz}$ band (Risacher et al., 2016a). A year later, in May 2016, the second frequency band, the high-frequency array upGREAT/HFA operating at $4.7 \mathrm{THz}$ was successfully commissioned. As the SOFIA project had only one flight compressor in 2015/2016, only one of the arrays could be operated at a given time. In 2017 , the phase 2 SOFIA infrastructure was completed, for the first time allowing two compressors, and therefore two closed cycle systems to operate independently and simultaneously. upGREAT in its full dual-array configuration was then commissioned in May/June 2017 and has been operated since during 25 science flights. This paper describes the development of the instrument and its in-flight performance.

\section{SOFIA Observations - Atmospheric Conditions}

Observations in the $\mathrm{THz}$ range are almost impossible to achieve from ground, therefore only airborne, balloon borne or space telescopes can perform such observations. For SOFIA, flying regularly at altitudes above 41,000 feet, a large part of this spectrum becomes accessible with high atmospheric transmission. Even though it flies above $99 \%$ of the Earth's atmosphere, it still suffers from absorption from several atmospheric molecular species, e.g. $\mathrm{H}_{2} \mathrm{O}, \mathrm{O}_{3}$. Figures 1 and 2 show the typical atmospheric transmission for the two upGREAT bands, for 1.8$2.07 \mathrm{THz}$ and around $4.745 \mathrm{THz}$. Some of the main lines of interest are shown overlaid. Figure 1 shows that a large part of the band is not accessible, and even in the regions having good atmospheric transmission, not all frequencies are tunable with the current local oscillators (LOs) (Sec. 4.2). For the higher frequencies, as can be seen in Fig. 2, the [OI] observations are very challenging to perform, even

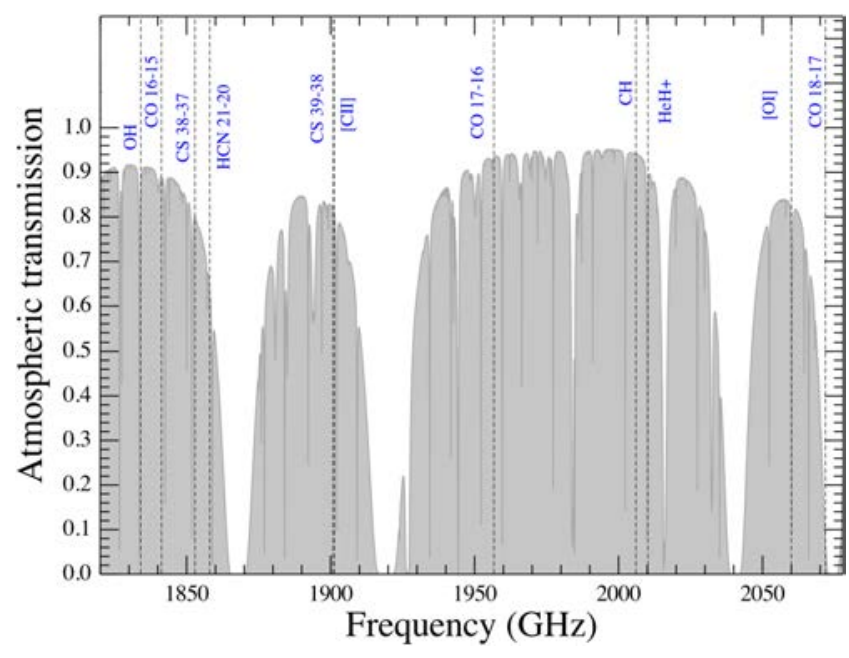

Fig. 1. Atmospheric transmission at 43,000 feet with $15 \mu \mathrm{m}$ water vapor.

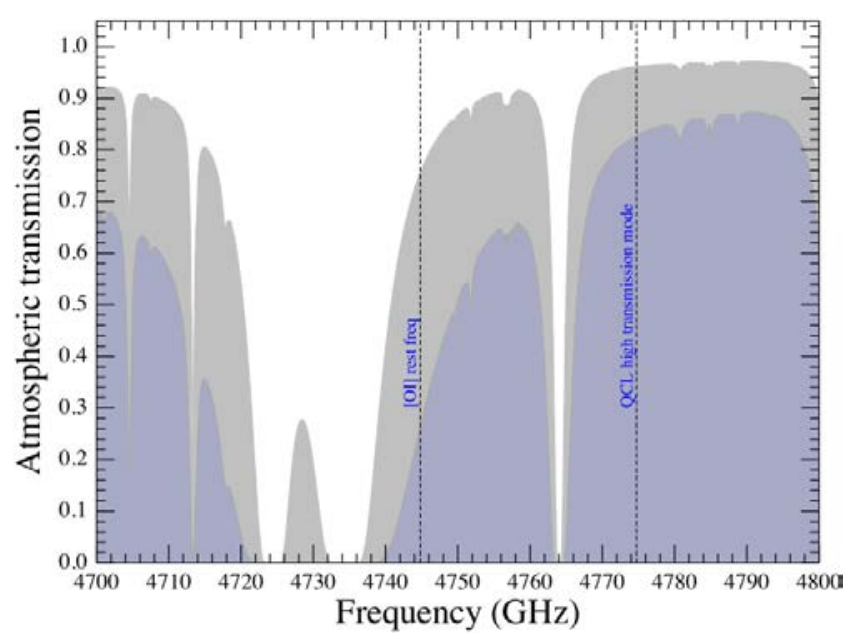

Fig. 2. Atmospheric transmission at 43,000 feet with $15 \mu \mathrm{m}$ water vapor (purple) and $5 \mu \mathrm{m}$ (grey). At the [OI] rest frequency, the atmospheric transmission more than doubles in this case.

from SOFIA, and require precipitable water vapors (PWV) well below $10 \mu \mathrm{m}$, in order not to be too degraded by the atmospheric losses. Such PWV values are difficult to achieve when SOFIA is stationed in Palmdale, CA, where it normally operates. However, SOFIA is deployed yearly to New Zealand and is stationed in Christchurch during the Southern winter. There, the atmospheric conditions are usually of the order of a few microns of PWV, hence allowing more efficient $[\mathrm{OI}]$ observations.

\section{Instrument General Description}

A full detailed description of the receivers hardware can be found in Risacher et al. (2016a) and the 
Table 1. upGREAT receiver characteristics.

\begin{tabular}{lccc}
\hline & LFA & HFA & Comment \\
\hline RF Bandwidth & $1.83-2.006 \mathrm{THz} *$ & $4.745 \mathrm{THz} \pm$ few GHz & $*$ up to 2.07 THz (experimental) \\
IF Bandwidth & $0.5-4 \mathrm{GHz}$ & $0.5-4.0 \mathrm{GHz}$ & \\
Spectral resolution & $\gtrsim 10^{7}$ & N $10^{7}$ & Waveguide-based \\
Mixer technology & NbN HEB & NbN HEB & SSB, at 2 GHz IF \\
LO technology & Solid state multipliers & $2500 \mathrm{~K}$ & Weather dependent \\
Receiver Sensitivity & $2000 \mathrm{~K}$ & $3500 \mathrm{~K}$ & FFTS technology \\
System noise temperature & $2000-2500 \mathrm{~K}$ & Seven in one sub-array & \\
Number of pixels & Seven per sub-array (14 total) & 4 GHz instantaneous BW & \\
Backends & $4 \mathrm{GHz}$ instantaneous BW & Hexagonal with central pixel & \\
Array Geometry & Hexagonal with central pixel & Hexagers & \\
\hline
\end{tabular}

first commissioning results of the low frequency upGREAT receiver are presented in Risacher et al. (2016b). We present here the instrument performance as of 2018, highlighting the performance of the high frequency array for $4.7 \mathrm{THz}$ operation, and describing the improvements and experience gained since the installation of those systems. Since May 2017, the two upGREAT arrays are operating in parallel, with 21 pixels. The main instrument characteristics are summarized in Table 1.

The main components of the upGREAT LFA/HFA configuration, are shown in Fig. 3. The astronomical signal is directed through the SOFIA telescope main optics and the first optical component from the GREAT system is the derotator optics, a "K-mirror" rotating set of mirrors, which allows to compensate for the sky rotation in the instrument focal plane (described in detail in
Risacher et al. (2016b)). The frequency selection is done via a dichroic filter from QMC Instruments Ltd., which separates in transmission the low frequencies below $3 \mathrm{THz}$ (to the upGREAT LFA system), and reflects the $4.7 \mathrm{THz}$ signal (to the HFA receiver) with less than $7 \%$ and $2 \%$ losses, respectively. This component allows the simultaneous operation of both upGREAT arrays. Then the LO references are combined with the astronomical signal, via dedicated pre-adjusted optics, which also reimage as needed the optical parameters via pairs of Gaussian telescope optics (details in Risacher et al. (2016a) and in following sections). The LO references are located inside elongated housings mounted under the Science Instrument (SI). For the upGREAT/LFA system, the LO systems are based on multiplier chains from VDI Inc. and for the HFA system, the LO system is using quantum-cascade

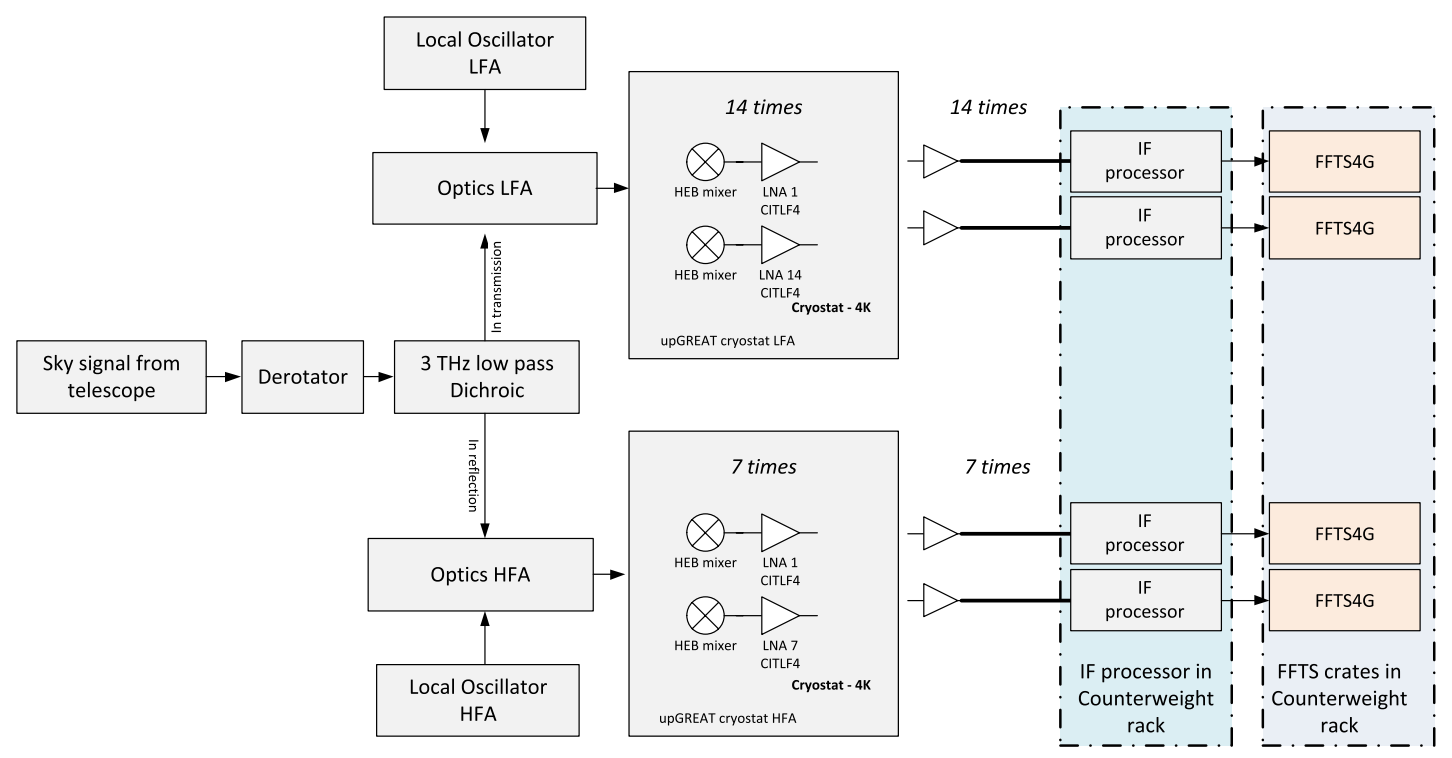

Fig. 3. Diagram showing the main components of the upGREAT receiver system-adapted from Risacher et al. (2016a). 
devices (detailed in Secs. 5.2 and 5.3). Details on the cryostat, mixer design, IF system and backends can be found in Risacher et al. (2016a). The superconducting Hot Electron Bolometers (HEB) mixers are developed and fabricated by the I. Physikalisches Institut der Universität zu Köln (Pütz et al., 2012; Büchel et al., 2015).

\section{LFA Array}

\subsection{Initial design and optics modifications}

Compared to the original design for the upGREAT/ LFA array, several things were modified since the first installation onboard SOFIA (Risacher et al., 2016a). The optics plate and the LO optics were redesigned. The first implementation of the LO optics used two separate LO chains, having output signals whose polarization is perpendicular to each other and combined through a polarizer grid, in order to propagate both LO signals on a nominal common axis. Then, on the main optics plate, another polarizer grid would divide them again while being combined with the corresponding RF signals for each polarization. When using one LO chain at a time, illuminating its corresponding subarray worked fine, but when operated in parallel, severe interferences between the two LO systems occurred. The solution was to decouple completely the two LO paths, keeping one on-axis (for the so-called $H$-polarization subarray), while the other LO had a tilted axis. Therefore, the whole LO and LFA optics plate was redesigned to accommodate this change, which proved to be successful as the strong interferences disappeared. Figure 4 shows the updated diagram of the LO chains.

\subsection{LO limitations and LO improvements}

The upGREAT/LFA receiver is designed to cover the $1.8-2.5 \mathrm{THz}$ range. As such, the HEB mixers and the optics are all designed to cover that frequency range. Currently, the upGREAT/LFA system is offered to the community in the 1.83 $2.006 \mathrm{THz}$ range. This frequency limitation comes from the LO technology. The VDI LO multiplier sources allow to cover a moderate region around

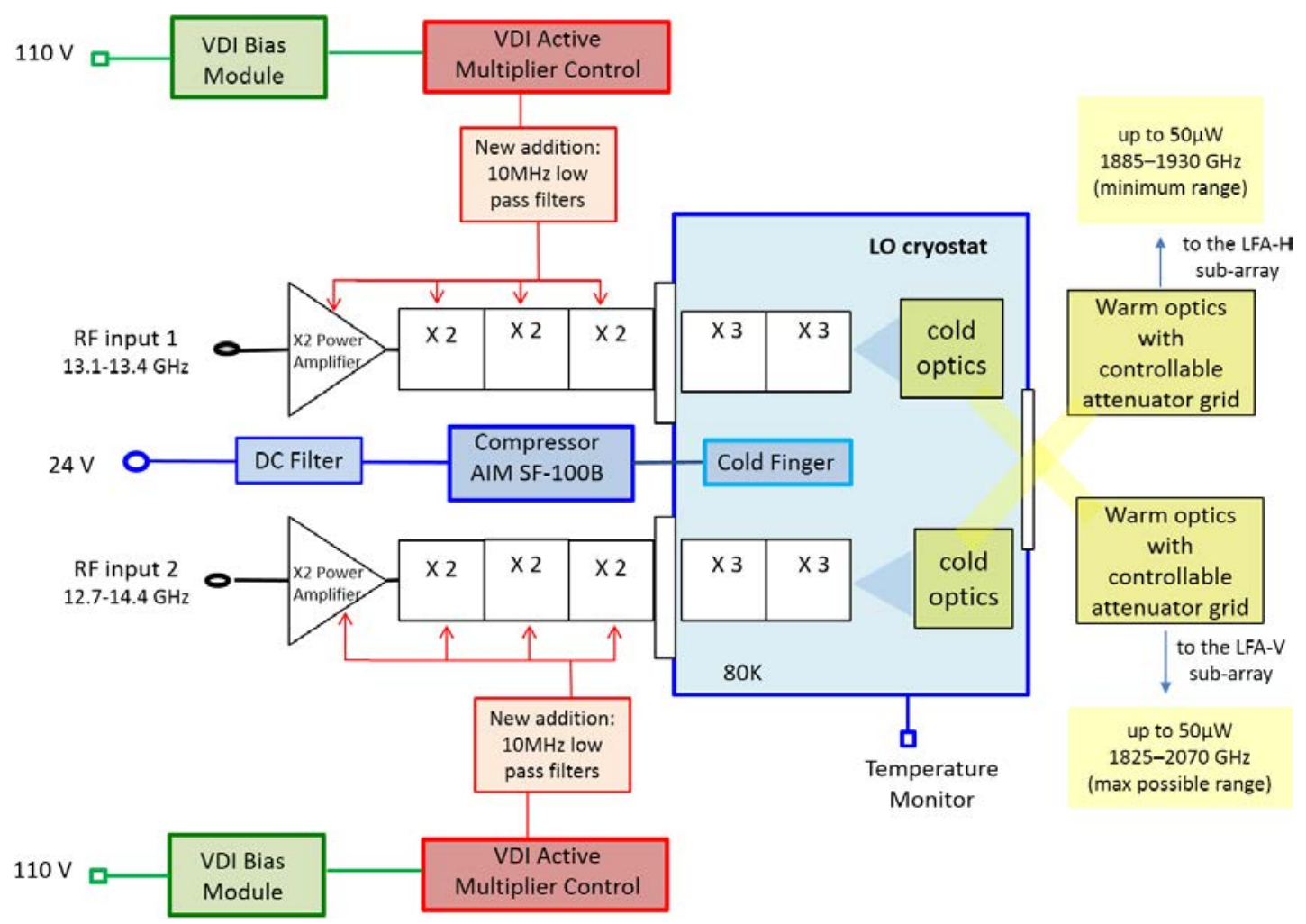

Fig. 4. Main LO diagram showing the modifications compared to the original configuration (Fig. 3 in Risacher et al. (2016a)). Each LO chain is now driven by independent synthesizers, the LO paths are now completely independent from each other and the output LO power adjustment is now performed by remotely controllable wire grids. Note also the inclusion of $10 \mathrm{MHz}$ low pass filters in every multiplier bias lines, allows to avoid spurious lines in the IF bandpass of the mixers. 
$1.9 \mathrm{THz}$ with very high output power. This development proved to be very challenging, and only the range $1.83-2.006 \mathrm{THz}$ could be covered with several LO AMC chains, and only one VDI system was capable of extending as far as $2.07 \mathrm{THz}$. This is especially important, as there are several astronomical lines of interest in that upper frequency range. Several attempts to replicate this performance with other AMC chains have not been successful until now. Therefore, observations in the $2.006-2.07 \mathrm{THz}$ range can only be offered through consortium flights, shared-risks, until more funding is allocated for more robust, additional LO sources.

Another major change was the redesign and rearrangement of the $\mathrm{LO}$ components inside the $\mathrm{LO}$ main enclosure. Indeed, it was found out that the thermal dissipation and air flow was not sufficient to adequately cool the main LO components, especially the power amplifiers from Spacek Labs. Each LO system generates a lot of power, $50 \mathrm{~W}$ at $26 \mathrm{GHz}$, $250 \mathrm{~mW}$ at $220 \mathrm{GHz}$, and $20-30 \mu \mathrm{W}$ at $1.9 \mathrm{THz}$ (or $40-60 \mu \mathrm{W}$ when the last two triplers are cooled down to $100 \mathrm{~K}$ ). They dissipate each $50 \mathrm{~W}$, then the Stirling cooler for the triplers also dissipates around $100 \mathrm{~W}$, therefore all in all, about $300 \mathrm{~W}$ are dissipated inside this small LO enclosure. Under normal laboratory conditions, at $20^{\circ} \mathrm{C}$, the power amplifiers case temperature would be around $35-40^{\circ} \mathrm{C}$. But once the instrument is installed onboard SOFIA, particularly during summer months in California's Mojave Desert, it has proven difficult to manage the cabin temperature in the hours leading up to takeoff. Cabin temperatures approaching $30^{\circ} \mathrm{C}$ in the aft cabin area can result in component instability and even failure. To improve this situation, components were rearranged, the air flow was improved, power amplifiers are now cooled conductively using heat sinks with cooling pipes together with more powerful fans. These changes resulted in $\sim 10^{\circ} \mathrm{C}$ lower temperatures in the LO enclosure. Though these design changes certainly improved the thermal overtemperature situation within the LO enclosure, the stability of these components is still susceptible to large cabin air temperature fluctuations in the first few hours of a flight.

There were also relatively strong interferences, spurs, present in many places in the RF band, originating from the LO chains. VDI has been optimizing and improving on the situation since 2015 . Several iterations of multiplier doublers have helped to clean up most of the band. A major breakthrough

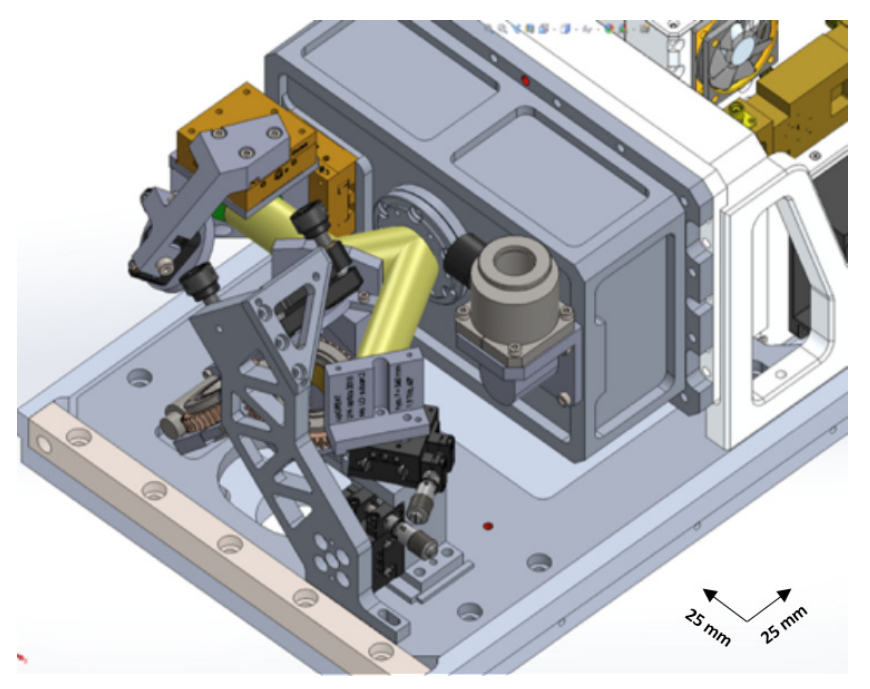

Fig. 5. Model showing the updated LO optics. The two LO chains are now fully independent, and their output paths are shown in yellow. Additional wire grids on motorized supports were added in February 2018, allowing to control the LO output levels of both polarizations independently.

in this regard occurred in 2017, when VDI identified a source of interference causing a forest of narrow spurs evenly spaced around $1 \mathrm{GHz}$ IF (at the IF output of the receiver). These narrow spurs had been present from the beginning for all pixels, and were flagged during data reduction. They originated from pickup from the multipliers bias lines of a small part of the input RF signal (via radiation). A redesign of this VDI electronic controller is ongoing, but the short term solution was to include $10 \mathrm{MHz}$ low pass filters for every multiplier bias line. This effectively solved the LO-based issues (while observations may still suffer from SOFIA-internal but also externally rooted RFI).

The most recent improvement performed in 2018 was the addition of LO attenuation wire grids, placed at the LO beam outputs (see Fig. 5), in order to independently adjust the LO levels for both subarrays. Before that, LO adjustment was performed electronically, or not done at all, therefore occasionally resulting in overpumped mixers, with some degradation in performance.

\section{HF A Array}

The second upGREAT array for high frequencies (HFA) was completed in mid-2016, and its commissioning started in November 2016. A view of the two closed-cycle receivers (upGREAT LFA+HFA) is shown in Fig. 6. 


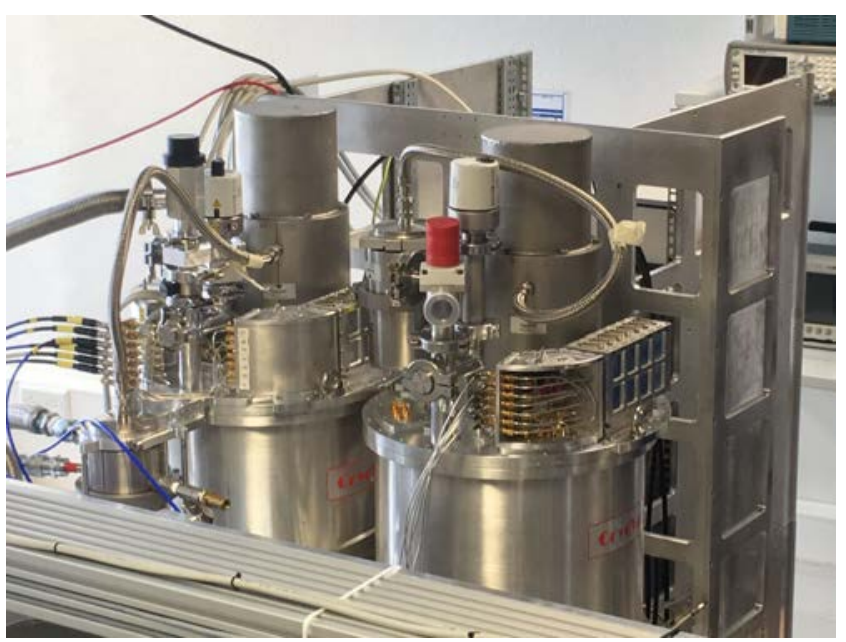

Fig. 6. View of the two closed-cycle receivers upGREAT LFA (left) and HFA (right).

\subsection{Initial design, optics}

As described in Risacher et al. (2016a), the cryostat was fabricated at CryoVac $\mathrm{GmbH}$, integrated with a transMIT pulse tube 2-stage cooler (PTD406C). The cryostat design is identical to the lower frequency array system (LFA), with some differences:

- Cryostat Window: $1 \mathrm{~mm}$ thick Silicon with Parylene-C coating and $525 \mu \mathrm{m}$ silicon with antireflection grooves (Wagner-Gentner et al., 2006)

- Infrared Filters: Originally a low pass $8 \mathrm{THz}$ filter from QMC was used at the $77 \mathrm{~K}$ stage, but it was removed as cooling power is sufficient. When removed, the impact on the cryostat and mixer temperatures was negligible. The loss at $4.7 \mathrm{THz}$ of that filter was of $7 \%$.

- Coupling optics: The optics of the upGREAT/ HFA are based on the optics design for the LFA. An assembly of six offset-parabolic mirrors in front of the six offset pixel HEBs forms the focal plane unit inside the cryostat. A dedicated elliptical mirror for the mixer of the center pixel is mounted separately behind this unit. Four active mirrors - common to all pixels and forming two Gaussian telescopes - map the cryostat focal plane to the focal plane of the SOFIA telescope. The cryostat houses one of these active mirrors, the other three are placed on the warm optics plate inside the optics compartment of the instrument structure. A first set of optical components was manufactured on an in-house Kern ultra-precision milling machine and are estimated to have a surface accuracy of a few microns RMS.
Switching from these bare aluminum mirrors to a commercially manufactured set of gold-plated mirrors with a specified surface accuracy of $10 \mathrm{~nm}$ did not notably improve the instrument performance. On the warm optics plate, the LO beam and the signal beam are superimposed by a thin Mylar foil (2.5 to $3.5 \mu \mathrm{m}$ thickness). A $3.3 \mathrm{THz}$ low-pass dichroic foil manufactured by QMC is used to split the HFA signal beam and the beam for the second installed GREAT channel. The optics are prepared for implementation of a second 7-pixel subarray for dual polarization observations.

\subsection{Mixers fabrication}

The HEB device fabrication, the feedhorns manufacturing and the subsequent integration of the mixer block proved to be challenging tasks. The feedhorns used are based on the smooth-wall spline horns (Granet et al., 2004) and are a scaled version of the LFA horns. They were fabricated by electroforming through Radiometer Physics, and they proved to be very difficult to fabricate with the required accuracy (low yield), mainly due to the difficult removal of the mandrel material inside the small waveguide section. Also in the block manufacturing in the Cologne workshop and especially in the assembly tolerances the approximate factor of 2 reduction in all dimensions compared to the LFA made a notable difference. More devices became damaged upon assembly and all mixers had to be pre-tested as single pixel in Cologne to ascertain their performance as a check on the assembly tolerances. The device fabrication was also not straightforward. As was done for the upGREAT/ LFA mixer junctions, the HEB film thickness was decreased in order to reduce the LO power requirement for the array. However, those reducedthickness devices did not perform as well as the original 4.7 THz single pixel HEB mixer that flew on SOFIA (Büchel et al., 2015). Also, it appeared that the extremely thin NbN films suffered more from environmental effects resulting in performance deterioration over several cooling cycles. Finally, as there is sufficient LO power with the QCL technology (see Sec. 5.3), the original HEB device was kept and the upGREAT/HFA devices are based on it, reproducing the previous single pixel performance. A cross-section view of the HFA mixer block and chip assembly can be seen in Fig. 7 . 


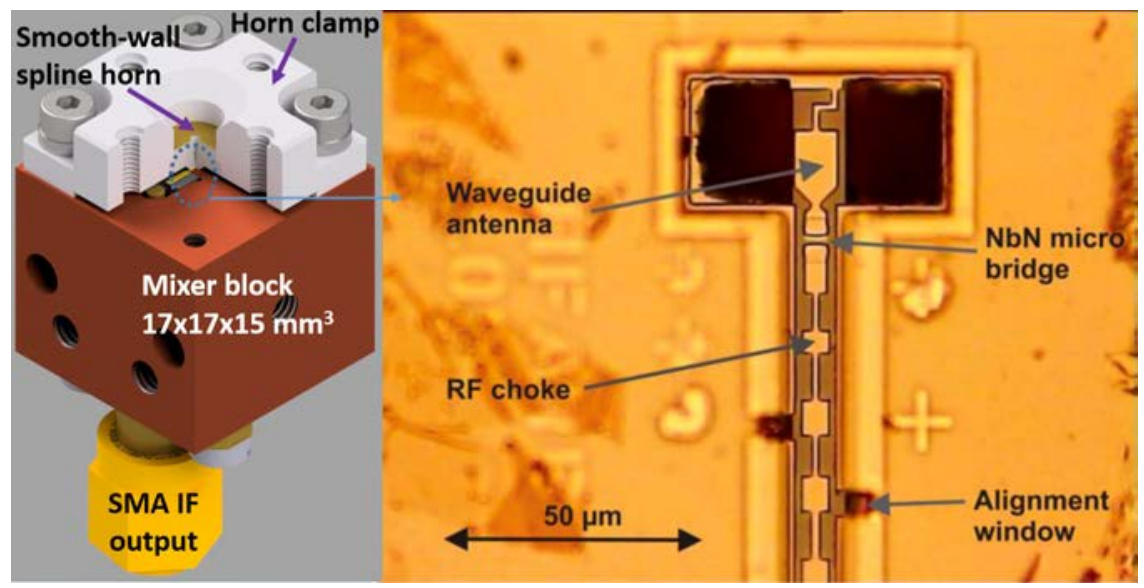

Fig. 7. Left: Cross-section view of one of the HFA mixer blocks showing the main components. Right: View of the mixer chip, showing the input waveguide, the waveguide antenna and the NbN HEB.

\subsection{QCL local oscillators}

The LO technology uses Quantum-Cascade Lasers (QCL). Two groups, the Institute of Optical Sensor Systems, German Aerospace Center (DLR) and the Physikalisches Institut der Universität zu Köln, (KOSMA hereafter) have carried out parallel developments, and both have succeeded in providing flightworthy hardware. The DLR QCL has been flying since 2016 with the HFA array, and the KOSMA QCL started flying since May 2018.

\subsubsection{DLR QCL LO evolution and performance}

The DLR QCL system (see Fig. 8) is a further development of the GREAT single pixel $4.7 \mathrm{THz}$ receiver, which had been successfully operated on

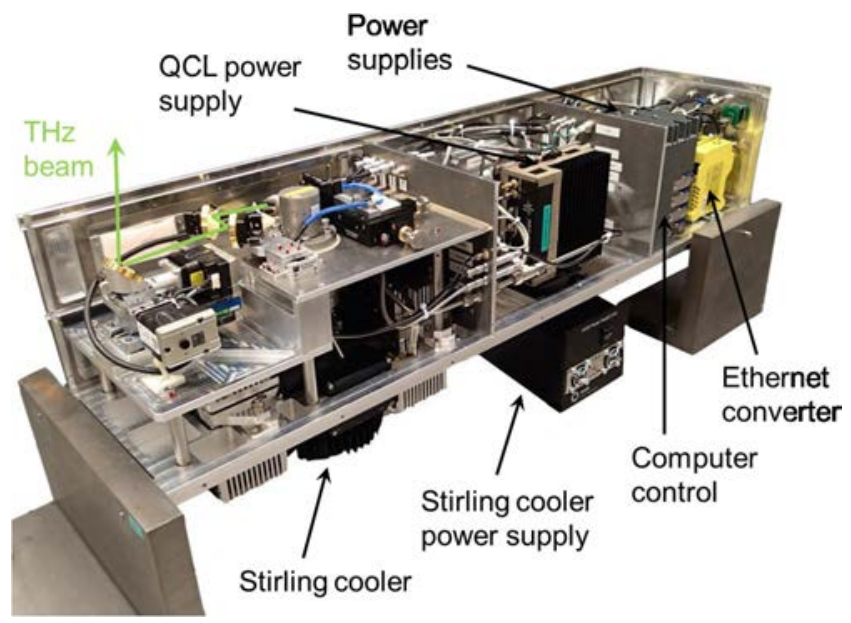

Fig. 8. Photograph of the opened LO box on a holder in the laboratory. Dimensions of the box are $100 \times 23 \times 23 \mathrm{~cm}^{3}$. The power supply for the Stirling cooler is the only external component which is needed for operation of the system. board of SOFIA from May 2014 to November 2016 (Richter et al., 2015). The QCL LO system consists of a $4.7-\mathrm{THz}$ QCL with a compact, low-input-power Stirling cooler (Ricor K535). The QCL is mounted inside a vacuum housing on the cold finger of the cryocooler, which provides a temperature between 30 and $80 \mathrm{~K}$, necessary for the operation of the QCL. No liquid cryogens are required. The active medium of the QCL is based on a hybrid design and has been developed for continuous-wave operation, high output power, and low electrical pump powers (Schrottke et al., 2013). Efficient carrier injection is achieved by resonant longitudinal optical phonon scattering. This design allows for an operating voltage below $6 \mathrm{~V}$. The amount of generated heat complies with the cooling capacity of the Stirling cooler of $7 \mathrm{~W}$ at $65 \mathrm{~K}$ with $240 \mathrm{~W}$ of electrical input power (Richter et al., 2010). The QCL has a lateral distributed feedback grating, which is optimized for the transition frequency of OI at $4.745 \mathrm{THz}$. Due to the DFB grating, the QCL has a single mode emission. The radiation is coupled out through one of the end facets of the single-plasmon waveguide and focused with a parabolic mirror. The beam profile is almost Gaussian with an $\mathrm{M}^{2}$ value of approximately 1.2. A flat mirror which is mounted on a goniometer allows aligning of the QCL-beam in order to direct it to a Fourier grating to multiplex the single beam into seven equal beams (see Sec. 5.4). The emission frequency of the LO is determined by measuring the absorption spectrum of $\mathrm{CH}_{3} \mathrm{OH}$ and comparing this with data from the literature. The LO covers a frequency range from -1.5 to $+6.5 \mathrm{GHz}$ around the rest frequency of $[\mathrm{OI}]$. The frequency is tunable by changing the laser 
driving current as well as by changing the heat sink temperature. At a constant heat sink temperature the frequency coverage of the $\mathrm{LO}$ is up to $4 \mathrm{GHz}$, for example at $52 \mathrm{~K}$ it ranges from -1.1 to $+2.6 \mathrm{GHz}$. In general, tuning of the frequency by current is preferred, because it is faster than tuning by changing the heat sink temperature, which requires more time, because thermal equilibrium has to be established. The heat sink temperature is kept constant within $\pm 1 \mathrm{mK}$ by a dedicated control loop. The frequency stability is about a few $\mathrm{MHz}$ (Richter et al., 2015). The output power varies with the laser driving current and with the heat sink temperature. For all LO frequencies it is above $1.2 \mathrm{~mW}$ with a maximum power reaching $2.2 \mathrm{~mW}$. This includes absorption loss by the window in the vacuum housing of the Stirling cooler, as well as atmospheric absorption loss. Corrected for these losses the output power of the QCL is up to $6 \mathrm{~mW}$. The LO system is fully controlled by a dedicated software which controls the Stirling cooler itself, the electrical input power of the QCL, and the heatsink temperature.

\subsubsection{KOSMA QCL LO performance}

The KOSMA QCL LO (see Figs. 9 and 10) relies on a single mode, double metal QCL with integrated patch antenna array (Bosco et al., 2016; Justen et al., 2016) delivering about $2.4 \mathrm{~mW}$ optical power in the fundamental Gaussian mode. About 25 to 30 percent of the power is needed to pump the 7-pixel HEB mixer array. The power can be varied by a polarizer grid in the HFA optics compartment. The

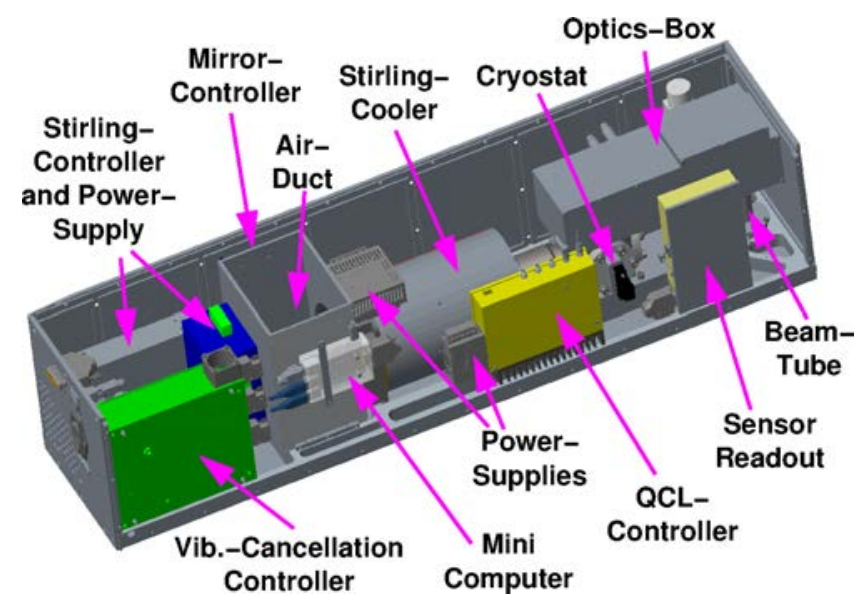

Fig. 9. KOSMA QCL LO box enclosure showing the main components. It has the same outer dimensions as the box shown in Fig. 8.

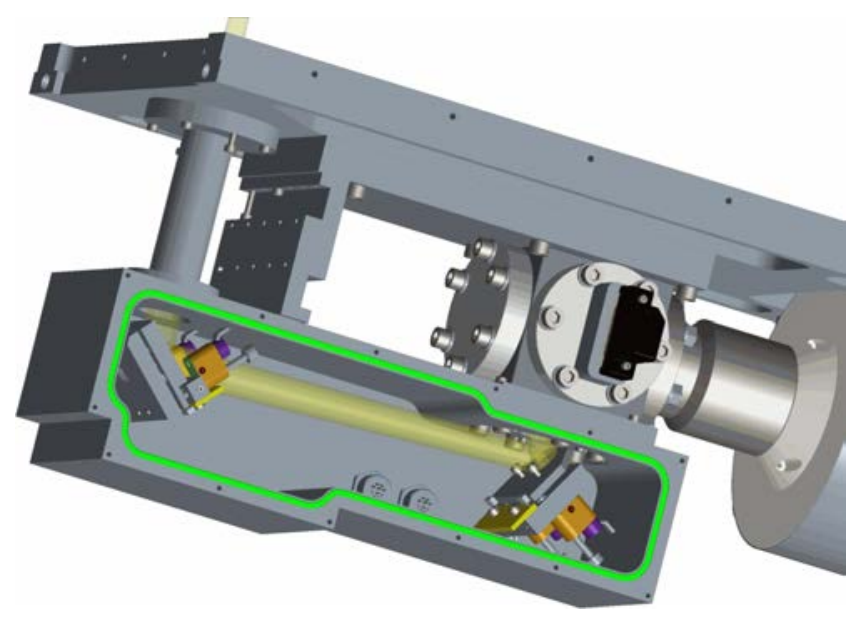

Fig. 10. The KOSMA QCL optics is located inside a hermetic box indicated by the green contour. This box is connected to the main instrument compartment via sealed fittings, without any RF windows (as opposed to all the other GREAT local oscillator boxes). This allows during flights, to have the QCL optics compartment at the ambient outside air pressure, about $160 \mathrm{mBar}$, therefore maximizing the QCL LO signal transmission.

QCL can be tuned from -2.5 to $+2.9 \mathrm{GHz}$ around the OI line at $4744.8 \mathrm{GHz}$ by controling the bath temperature to a few $\mathrm{mK}$ between 40 and $70 \mathrm{~K}$. The QCL is cooled by a Stirling cryocooler. No liquid cryogens are required. The beam position and beam angle are adjusted with two tip-tilt mirrors which couple the beam into the HFA optics compartment. In order to avoid absorption of the LO signal by water in the surrounding air, the beam path is contained in a hermetic housing, which is open to the inside of the instrument optics compartment and therefore experience the same external air pressure (typically about $160 \mathrm{mBar}$ ) during flight. The LO system is controlled remotely over an Ethernet connection by a dedicated computer which controls the Stirling cooler, vibration control, QCL current source, QCL temperature control and mirror positions. A prototype of this QCL was used in Cologne to pre-test the $4.7 \mathrm{THz}$ mixers in a single pixel set-up.

\subsubsection{QCL LO frequency stability}

Currently, the QCL LOs are free-running and their absolute frequency stability is set by the temperature and current stability of the lasers. For the upGREAT/HFA observations, the [OI] frequency scale is established by referencing to the telluric [OI] that is prominently present in all data, to a precision of better than $0.1 \mathrm{~km} / \mathrm{s}$, however this requires 

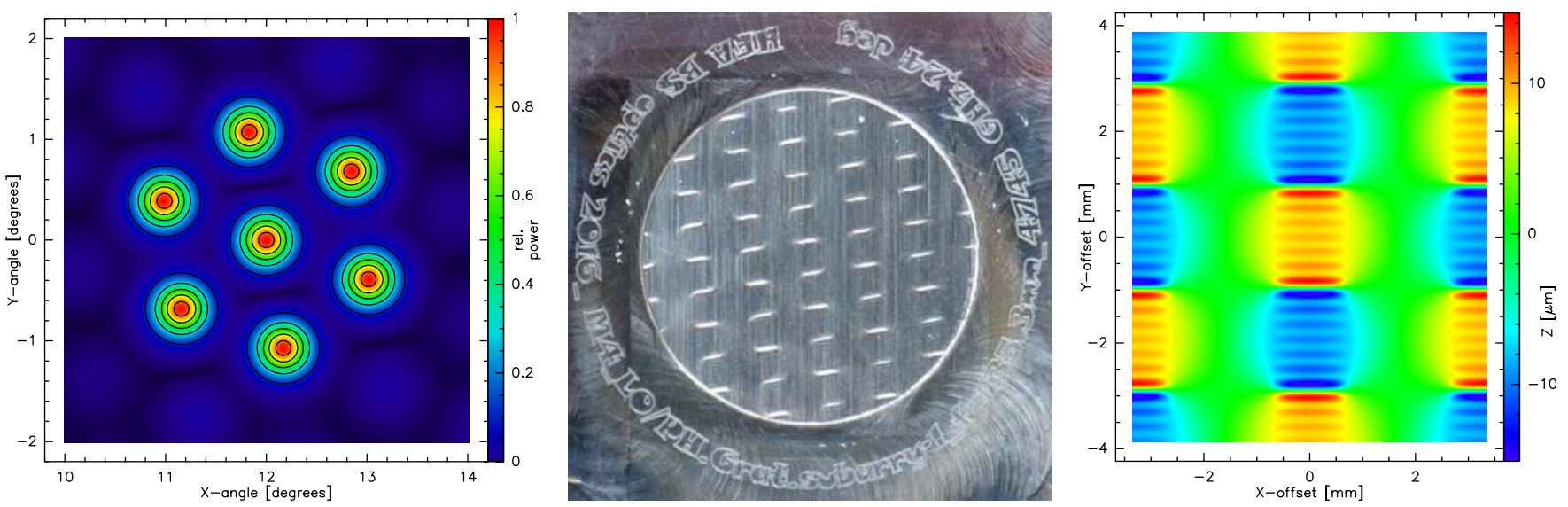

Fig. 11. Collimating Fourier grating operating at $4.7 \mathrm{THz}$ : calculated diffraction pattern (left), photograph of the final grating (center) and surface structure (right). We show two unit cells of the grating surface to illustrate its hexagonal symmetry.

additional post processing steps in the data reduction. For the DLR QCL, the estimated linewidth is of a few $\mathrm{MHz}$ and drifts can be of the same order. When using the KOSMA QCL, measurements of the beat signal between the QCL and a microwave source showed that the QCL frequency jitter is smaller than $1 \mathrm{MHz}(0.05 \mathrm{~km} / \mathrm{sec})$. Similarly, the laser frequency drift during observations, as measured by comparison with the telluric OI absorption line is also better than $1 \mathrm{MHz}$. Several options are under study to achieve a frequency/phase stabilization of the QCL, the main obstacle for this being the limited physical space available in the instrument to place additional components.

\subsection{LO distribution}

For the LO distribution, as in the LFA array, one single LO source is equally multiplexed into seven beams via a phase grating. This collimating Fourier grating (CFG), distributes the LO signal to the array elements (Heyminck \& Graf, 2001). The grating structure (shown in Fig. 11) is the same as in the LFA band (Risacher et al., 2016a) or in the CHAMP + receiver (Kasemann et al., 2008). The nominal efficiency ${ }^{(a)}$ of the grating is approximately $90 \%$. The RMS power imbalance between the seven beams is ideally less than $0.5 \%$ at the designed frequency. The CFG has a diameter of $20 \mathrm{~mm}$, a focal length of $135.3 \mathrm{~mm}$ and operates at a reflection angle of $24^{\circ}$. In order to comply with optomechanical boundary conditions, the grating

\footnotetext{
${ }^{\mathrm{a}}$ Calculated as the fraction of power contained in the desired diffraction orders relative to the total power in the diffraction pattern.
}

structure is designed to produce a diffraction pattern rotated by $9^{\circ}$ with respect to the reflection plane.

\section{Instrument Installation Onboard SOFIA}

This section describes the setup and typical installation procedure of the SI into the aircraft. The various SOFIA SIs are connected to a main reference, the SI flange, which is at the end of the Nasmyth Tube. This is inherited from the Kuiper Airborne Observatory (KAO), SOFIA's predecessor, with a 36-inch diameter primary mirror on a Lockheed C-141A platform which was operational from 1975-1995. Typically, an instrument is installed aboard SOFIA and stays there for a flight series lasting from one to several weeks. Figure 12 shows the GREAT instrument with the upGREAT arrays on the aircraft just before the final installation and connection to the SI flange.

After the SI is connected mechanically, all of the electrical connections are done and tested. Typically, as the instrument has been undergoing testing in the laboratory facilities before the installation, it is already cold. The helium lines are connected from the instrument to the patch panel, and the compressors are started as soon as possible to resume its cooling. As long as the SI installation can be done in under $2 \mathrm{~h}$, this procedure allows adequate time for thermal recovery of the closed-cycle systems, resulting in an operational system within half a day. Figure 13 shows the typical work configuration during ground testing or flights.

\subsection{Cryocoolers onboard SOFIA}

The cryocooler system is permanently installed as part of the observatory on SOFIA and includes 


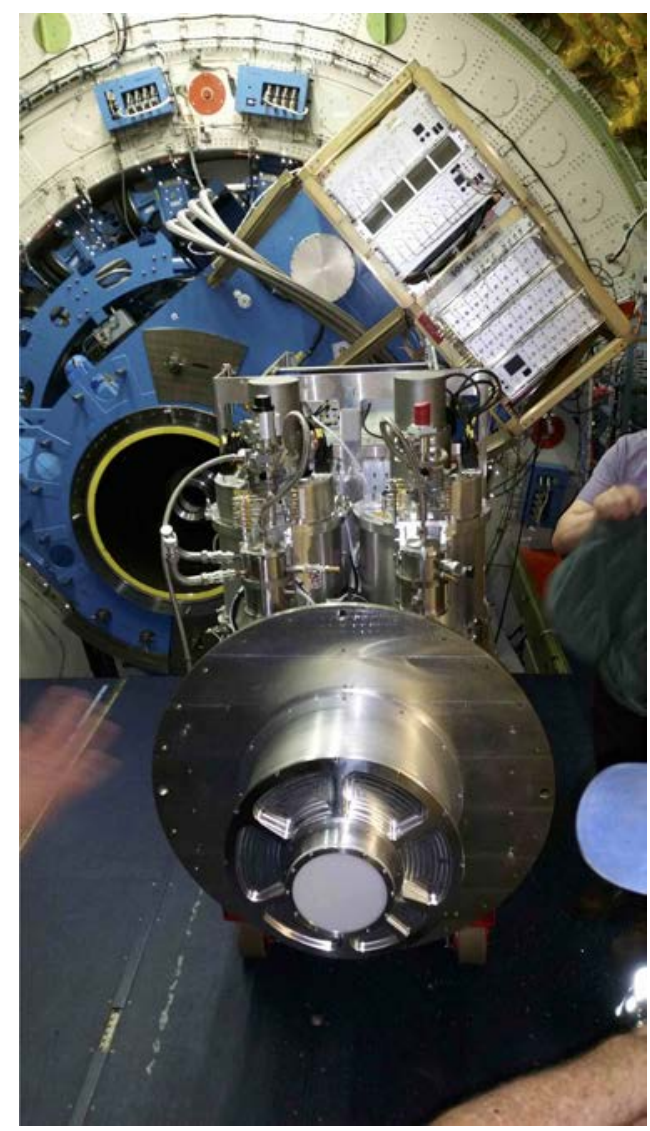

Fig. 12. Science Instrument before being turned by $180^{\circ}$ and connected to the interface flange (with the yellow ring). The HDPE window visible at the front of the instrument is removed before the final connection (only used for laboratory characterization to allow pumping the optics compartment). components located primarily on the aft upper deck but with a remote control panel located in the main cabin. The cryocooler system provides closed-loop cryogenic cooling for SIs with the following benefits:

- Continuous cooling of the instrument as opposed to using expendable liquid cryogens, which typically have $\sim 24 \mathrm{~h}$ hold time.

- Eliminates material handling hazards and cost of expendable cryogens.

- Simpler maintenance, operations, and logistics by eliminating the need to refill cryogen reservoirs.

The cryocooler system capable of driving two cold heads, completed Integration and Test (I\&T) and Verification and Validation (V\&V) in 2017 (overview shown in Fig. 14, photograph in Fig. 15). It consists of two ruggedized Commercial Off The Shelf (COTS) liquid-cooled scroll Cryomech compressors that use helium as an operating fluid to cryogenically cool the SI. Operation of the cryocooler is largely automatic, with two modes, a ground mode and flight mode that determine where and how the waste heat from the compressor(s) is rejected. The cryocooler has automatic monitoring and is designed to shut itself off in the event of critical faults. The cryocooler draws about $23 \mathrm{kVA}$ from the observatory bus 4, $115 \mathrm{VAC}, 400 \mathrm{~Hz}$, three-phase power source, when both compressors are operating at maximum load. Eight frequency converters in the aft upper deck convert the input

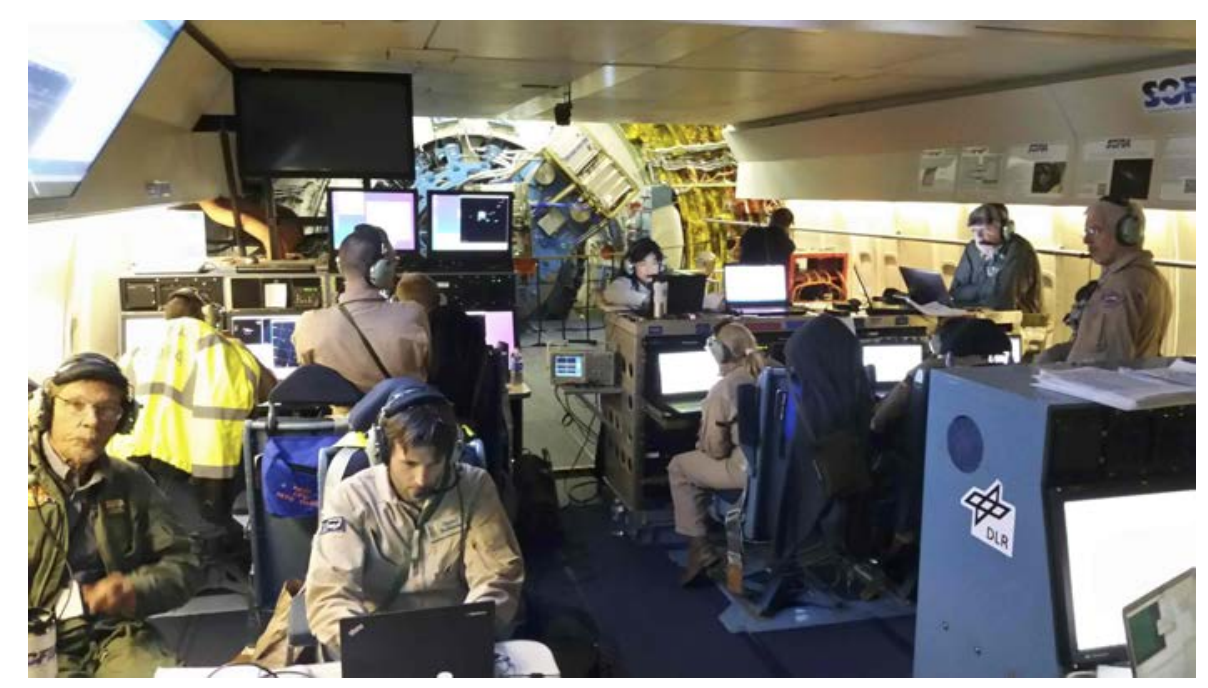

Fig. 13. Observers onboard SOFIA during a flight using the GREAT instrument. The SI is located at the far end (the counterweight rack with its instrumentation can be seen at the aft end of the pressurized main cabin). The instrument team can be seen on the right side of the photograph, tuning the instrument, sending the observing commands to the telescope and analyzing the collected data in realtime. On the left of the picture, the telescope team ensures the pointing of the telescope using its various star trackers, and monitors the good operation of the telescope during the observations. On the far right side, a small portion of the mission director (MD) console can be seen. The MD is responsible for coordinating all the teams together, the instrument team, the telescope team and the pilots. 


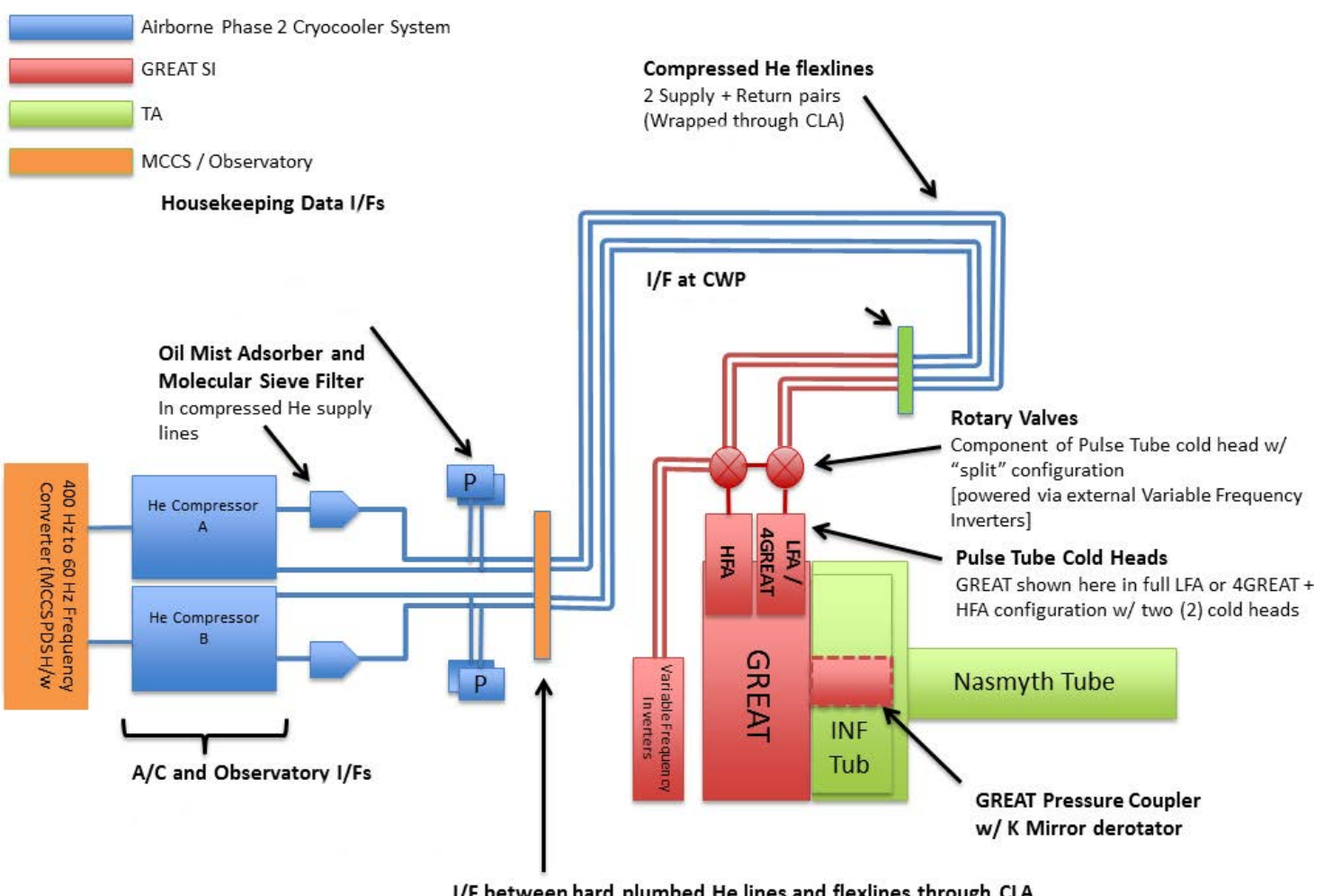

Fig. 14. Schematic showing the SOFIA cryocooler infrastructure.

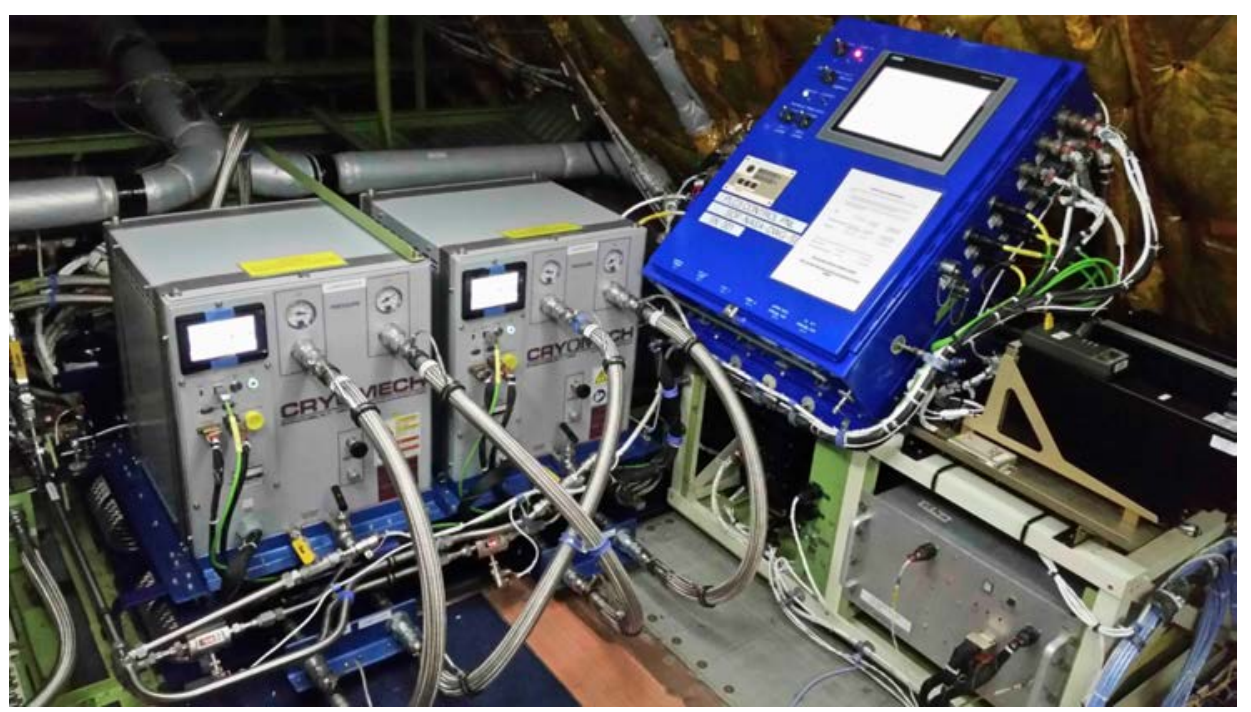

Fig. 15. Compressors located on the upper deck of the SOFIA Boeing 747.

power from $400 \mathrm{~Hz}$ to $60 \mathrm{~Hz}$ for the compressors, coolant pump and controller operation. The two heat exchanger axial fans operate from unconverted $400 \mathrm{~Hz}$, three-phase power from observatory bus 4 .

The cryocooler power source is downstream of the cabin depressurization relay which will remove power from all components in the event of depressurization.

To maintain ideal SI detector temperature, the cryogenic cooling system should operate continuously, although short (30-45 min) interruptions are tolerable and occur regularly when the plane is 
towed between the hangar and the airfield, or during maintenance.

\section{Performance on Sky}

The performance of the upGREAT/LFA system was already shown in Risacher et al. (2016b). We present here some results for the upGREAT/HFA system, taken during flight campaigns in 2017 and 2018.

\subsection{In-flight instrument performance verification}

\subsection{1. upGREAT/HFA receiver sensitivities and stability}

The upGREAT/HFA best achievable sensitivity can be seen in Fig. 16. The left plot shows the HEB mixers IV curves for the best LO distribution between pixels. The mixers achieve their optimum performance only over a narrow range in LO power, which is not identical for all pixels. The right plot shows the measured double sideband receiver noise temperature over its IF range with bias settings of $1.5 \mathrm{mV}$ for all pixels. For projects requiring best stability, different bias settings are recommended, with slightly degraded sensitivities. During normal observations, the time allocated for tuning optimizations can be less than a few minutes, therefore there is generally insufficient time to ideally set the parameters (the most critical one is the LO power distribution between the various pixels) and achieving the performance shown in Fig. 16. As a consequence, some of the pixels can have degraded performance. Thus, for each flight leg, the project scientist has to trade-off between observing time and best possible performance.

To assess the instrument stability performance, a typical figure-of-merit is the Allan variance. The results for the upGREAT/LFA receiver were detailed in Risacher et al. (2016a), in Sec. IV.B.4. Under best stable ambient temperature conditions, the spectroscopic stability between $1.4 \mathrm{MHz}$ width channels is typically above $80-100 \mathrm{~s}$ (Allan time). But if ambient temperature is not stable and drifts for example more than 1 degree per hour, the stability can easily degrade to $30-40 \mathrm{~s}$. For the upGREAT/HFA receiver, performance is similar, only slightly worse, with spectroscopic Allan times typically better than $60 \mathrm{~s}$. It also appears that the upGREAT/HFA system is more sensitive to the pulse-tube induced mechanical vibrations, which modulate the LO coupling causing bandpass changes and ultimately the baseline can be dominated by the standing wave originating from the mismatch between the HEB mixers and the cryogenic amplifiers. There are several ways to mitigate this known effect. For chopped observations, data can be readout synchronously with the wobbler phase, effectively suppressing this effect. With this solution in
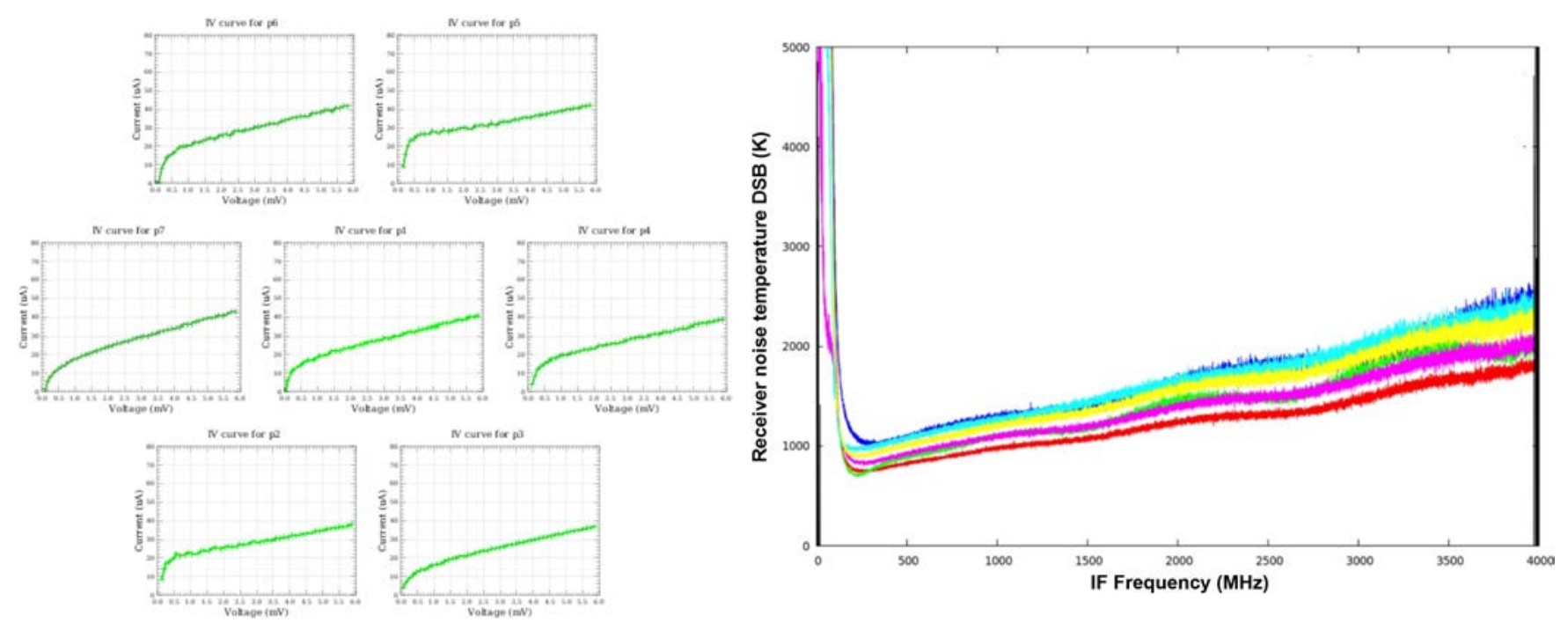

Fig. 16. Left: IV curves with LO power showing evenly balanced LO power for all pixels. Right: Double sideband noise temperature for the seven pixels when pumped under the best conditions (black and red curves are on top of each other). This represents the best achievable performance. Under normal flight conditions, as there is little time for tuning optimizations, the performance degrades for some of the pixels. 
chopped mode, no degradation or limitation due to this effect is seen in the baseline quality up to integrations of $\sim 2 \mathrm{~h}$ (more is difficult to accommodate with an airborne observatory). An example is given in Fig. 17 showing the typical achieved baseline quality.

If total power observations are requested (e.g. on-the-fly modes, up to $20 \mathrm{~s}$ on phase), procedures have been developed to minimize this baseline modulation during post-processing (Higgins, in prep.). This takes advantage of the fact that the spectral pattern is constant for a given tuning (pixel-based) and hence can be fit to observations of "blank" sky (an approach similar to what has been done with Herschel/HIFI in Kester et al. (2014)).

\subsubsection{Array parameters determination}

The necessary steps to be performed in the very first flight after (re-)installation of the instrument (or after a change of its configuration that might affect the optical alignment) are described for the LFA in Risacher et al. (2016b). For the upGREAT LFA+HFA characterization, we basically follow the same procedures, with parameters adjusted to the smaller (HFA) beam and the more compact array configuration:

- determine the instrument focal plane geometry (boresight and array geometry).

- determine the optimal focus position.

- measure the beam coupling (efficiencies) on a known calibrator.

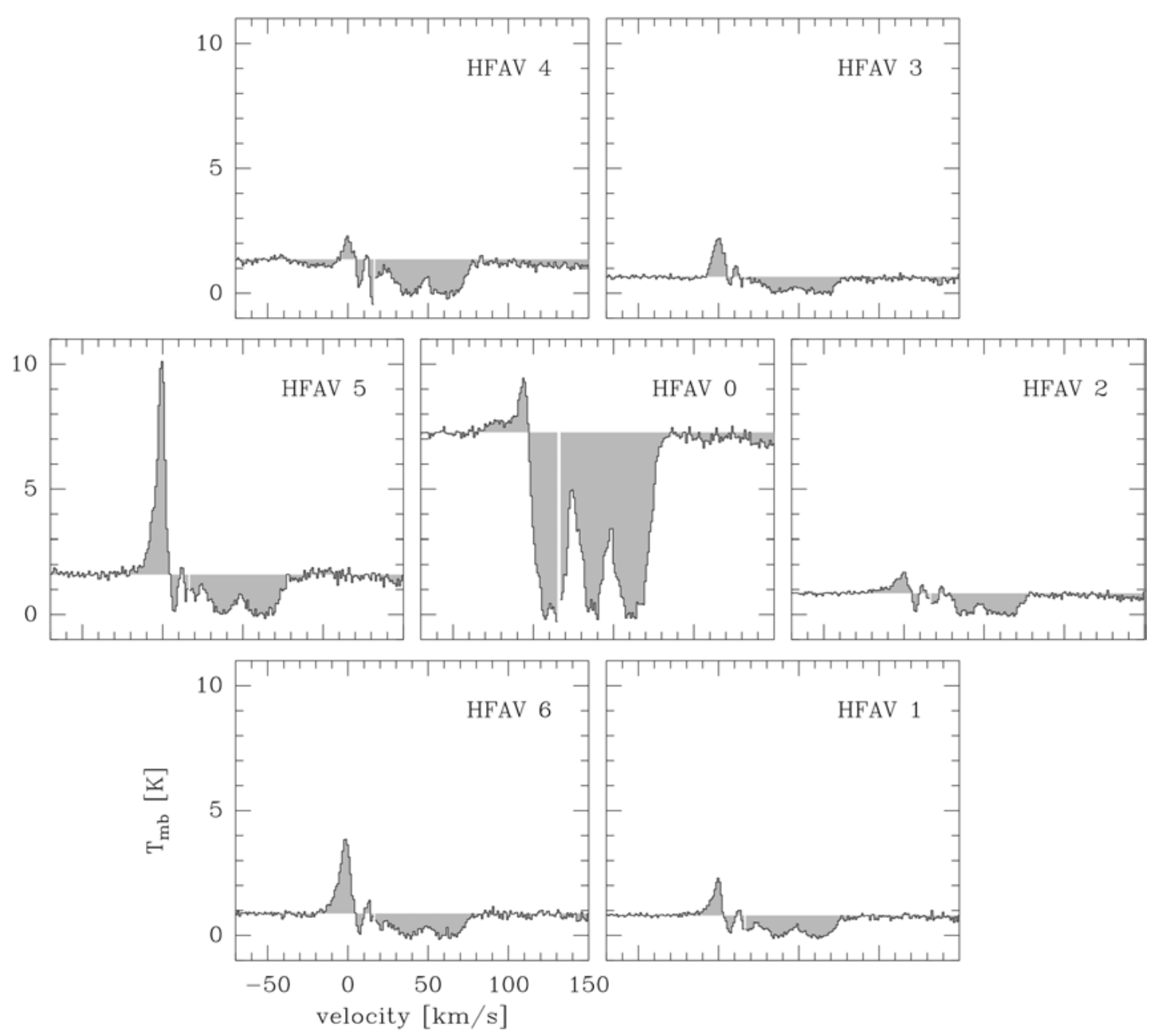

Fig. 17. OI $63 \mu \mathrm{m}$ spectra observed with the upGREAT/HFA towards the high-mass star forming core W49N, showing emission and strong absorption along the line-of-sight towards the prominent dust continuum emission of the source. The data was acquired on SOFIA flight 420 out of Christchurch, New Zealand, on July 15, 2017, during a $1.2 \mathrm{~h}$ flight leg at an altitude of $43 \mathrm{kft}$. Observations were performed in double-beam chopped mode, wobbling the subreflector at $2.5 \mathrm{~Hz}$ with a throw of $180^{\prime \prime}$. No baseline was removed, the spectra were box-smoothed to a spectral resolution of $1 \mathrm{~km} / \mathrm{s}$. 
The first step is to determine the arrays geometry in the focal plane and hence their optical alignments to the optics of the telescope's imager, which later on will control the tracking of the telescope (and hence the pointing of the GREAT instrument). Once the array geometry has been derived, the actual sky coordinate of each pixel will be introduced to the raw data header of each observation dump (typically every $0.3-1.0 \mathrm{~s}$ ). The alignment of, in particular, the HFA array (means, determining the pixel positions precisely) is challenging even on the brightest calibrators, requiring excellent residual atmospheric transmission. As an example, during our first and only flight out of Palmdale after reinstallation for the OC6-G series on May 22nd, 2018, flight planning enforced observations of Jupiter starting at low flight altitudes (36 kft) and mediocre atmospheric conditions $(15 \mu \mathrm{m}$ PWV). Short scale atmospheric instabilities (see Fig. 2 for a display of the limited atmospheric transmission near the [OI] line) made it impossible to derive the HFA parameters, and only with our first southern deployment flight (cruising now at $41-43 \mathrm{kft}$ in cold southern skies), the array parameters could be derived. From two observations of Mars in June 2018 (on June 16th, the diameter of the planet was $\left.18.9^{\prime \prime}\right)$, we determined the offset and angle of the rotator axis and the array geometries. We derive the individual pixel positions with an $\mathrm{rms}$ deviation of typically 1 ". The arrays' central positions were coaligned with respect to the central HFA pixel to $0.3^{\prime \prime}$ (LFAV) and 1.0" (LFAH), so well tolerable within their 14.1" main beam. In practice, for almost all projects we "tracked" on the central pixel of the HFA because of the higher tracking requirement of its smaller beam.

With extended Mars, the HFA beam sizes could not be well constrained during this year's observations but as the internal array optics has not changed since the commissioning of the individual arrays in 2016 (when Mars was more compact, $7.6^{\prime \prime}$ ), it is safe to adopt the beam sizes determined from those observations. On June 26th, 2018, we derived the coupling efficiencies of the LFA and HFA arrays with dedicated cross-scans of the individual array pixels across Mars. In Table 2, we quote the average main beam efficiency per subarray, with the standard deviation.

There are several science applications for which the absolute pointing accuracy of the system (telescope plus instrument) is critical. In some cases of observations towards dark cloud complexes (a prominent example is IRAS16293) where there are no tracking stars available for the telescope's focal plane imager and positioning based on gyroscopes and the wide field imagers may be unstable, drifting on short timescales to as much as $3-5^{\prime \prime}$, which is unsuitable for observations with a $6^{\prime \prime}$ beam. Under nominal circumstances, with a bright star in the FPI field, optical tracking is good to 0.5 arcsec. Tracking the instrument on the central HFA pixel, additional uncertainty comes from the precision of the determinations of the rotator offset and axis and of the pixel's offset from the rotator axis. The combination of these errors is obviously difficult to predict, but experience has shown that achieving an absolute positioning of HFAV00 to better than 1.5" is challenging.

\subsection{Science use/operation}

Since mid-2017 GREAT is operated in two configurations only: upGREAT/LFA with HFA and upGREAT/HFA with 4GREAT (Duran et al., in prep), thereby simplifying the operation and minimizing the requests for configuration change, without compromising on the frequency coverage. Since first light on April 01, 2011, GREAT has logged 137 science flights with more than 1000 successful science hours - including 63 flights with upGREAT/ LFA, 39 with upGREAT/HFA (25 flights in their joint configuration, since June 2017). More than 200 science projects have been successfully supported. The spatial multiplexing of the arrays now makes it possible to efficiently address large-scale mapping projects within a reasonable investment of

Table 2. upGREAT beam parameters (as determined on Mars in 11/2016 and 06/2018).

\begin{tabular}{lcc}
\hline Parameter & LFA $(1.9 \mathrm{THz})$ & HFA $(4.74 \mathrm{THz})$ \\
\hline Half power beam width & $14.1^{\prime \prime}$ & $6.3^{\prime \prime}$ \\
Array geometry: pixel spacing & $31.8^{\prime \prime}$ & $13.6^{\prime \prime}$ \\
Co-alignment of center pixel wrt. to HFA-V & $0.3^{\prime \prime}(\mathrm{V}), 1.0^{\prime \prime}(\mathrm{H})$ & \\
beam efficiency (Martian disk: $\left.18.9^{\prime \prime}\right)$ & $0.65(\mathrm{H}) / 0.66(\mathrm{~V})$ & $0.64(\mathrm{~V})$ \\
\hline
\end{tabular}




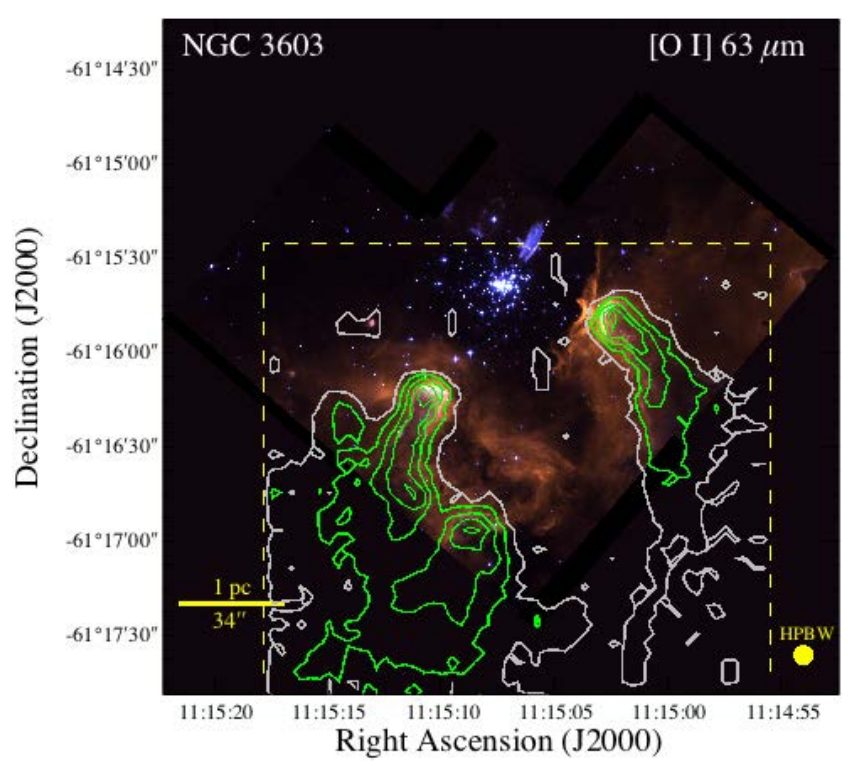

Fig. 18. The figure shows the (background) HST false color image (F656N-Red, average of F656N and F658N-Green, and F658N Blue from Brandner et al., 2000) of a $5 \times 4 \mathrm{pc}^{2}$ region around the NGC3603 star cluster. Discovered almost 200 years ago by John Herschel as a remarkable nebulosity in the constellation Carina, only studies in the last decades have revealed NGC3603's hidden nature as the Milky Way's most active stellar nursery. This densely packed condensation of young stars, with an equivalent mass of a few 10,000 suns in a volume with a diameter of only three light-years across, contains some of the most massive stars known. Their violent winds and ultraviolet radiation have cleared a cavity in the surrounding cloud that now allows unobscured views of the cluster. Deeply embedded in the extended cloud of gas and dust that gave birth to the visible cluster some 1 million years ago, the next generations of stars are forming already. Still invisible in the optical, these sites of ongoing star formation shine bright at farinfrared wavelengths and were the subject of study for GREAT during SOFIAs southern deployments (NGC3603 is visible only from southern skies). The contours correspond to the velocityintegrated intensity maps of the [OI] 63-micron fine-structure line obtained with the HFA, with a half-power beam width of $6.3^{\prime \prime}$. The region of the GREAT map matching the HST image is indicated with dashed lines. The peak intensity is $348 \mathrm{~K} \mathrm{~km} / \mathrm{s}$ (with contours in steps of $70 \mathrm{~K} \mathrm{~km} / \mathrm{s}$ from the peak). The picture was kindly provided by J.-P. Perez-Beaupuits, private communication.

observing time. In Fig. 18, we present an example of a large-scale [OI] $63 \mu \mathrm{m}$ fully-sampled map towards the prominent NGC3603 star forming region, covering an area of $160^{\prime \prime} \times 150^{\prime \prime}\left(4.7 \times 4.4 \mathrm{pc}^{2}\right)$. The map is composed of 2300 spectra, acquired in chopped on-the-fly mode (see Risacher et al. (2016b) for the description of the GREAT observing modes) during a short 45 min observing leg in summer 2017 (with excellent atmospheric conditions at $43 \mathrm{kft}$ flight altitude). The sensitivity across the map is very uniform, the noise rms of the data cube (in the velocity channels free of emission) is $2 \mathrm{~K}$ (with a spectral resolution of $0.5 \mathrm{~km} / \mathrm{s}$ )

Several legacy-type projects have been completed/launched with upGREAT during the last two years: e.g. a 1-square degree region of the Orion Molecular Cloud (Pabst et al. submitted), a fully-sampled map of the M51 galaxy (PIs: J. Pineda, J. Stutzki) is nearing completion. This year the distribution of $[\mathrm{CII}]$ and [OI] across the Central Molecular Zone $\left(1.5 \times 0.33 \mathrm{deg}^{2}\right.$, composed of 2.3 million spectra) (PIs: A. Harris, R. Güsten) has been measured during the southern deployment. None of these projects would have been possible without the enhanced mapping speed of upGREAT. All of these examples, together with future legacy projects that will be selected from the explicit calls for legacy proposals starting with observing cycle 7 , will contribute to the GREAT legacy of the SOFIA project.

\section{Future Work}

The main current limitation for the upGREAT/ LFA is the LO system. The solid state multiplier chains provide just enough output power, but are narrow in frequency range and quite fragile. A QCL option would provide much more output power but they are even more narrow band, and having a frequency locked system is not trivial. When the technology allows covering much more bandwidth, with enough output power, the entire range of the 1.8-2.5 THz could be covered at once.

The next natural step for the upGREAT/HFA array would be to populate its second polarization with seven additional pixels (currently on hold due to lack of funding). The cryostat is already prepared for it and has all the wiring in place. What is needed therefore is to fabricate seven new mixer blocks with the feedhorns. The LOs have enough power to pump 14 pixels. Currently, less than $5 \%$ of the LO power is coupled to the mixers, therefore $95 \%$ of it is being terminated into an absorber. It will make sense to use this $95 \%$ of LO power for the second polarization. Another future improvement will be to frequency-lock, or better yet, phase-lock the LO, in order to achieve a better frequency accuracy.

\section{Acknowledgments}

We thank the SOFIA engineering and operations teams whose support has been essential for the 
successful installation and operation of the upGREAT instrument. We thank the SOFIA project office at DLR, and in particular A. Himmes and D. Lilienthal for their outstanding continuous support during the development years of GREAT. We are grateful to J.-P. Perez-Beaupuits for kindly providing upGREAT results (Fig. 15) prior to publication. The development of upGREAT was financed by the participating institutes, by the Federal Ministry of Economics and Technology via the German Space Agency (DLR) under Grants 50 OK 1102, 50 OK 1103 and 50 OK 1104 and within the Collaborative Research Centre 956, sub-projects D2 and D3, funded by the Deutsche Forschungsgemeinschaft (DFG).

\section{References}

Bosco, L., Bonzon, C., Ohtani, K. et al. [2016] Appl. Phys. Lett. 109, 201103.

Brandner, W., Grebel, E. K., Chu, Y. H. et al. [2000] Astron. J. 119, 292.

Büchel, D., Pütz, P., Jacobs, K. et al. [2015] IEEE Trans. Terahertz Sci. Technol. 5(2), 207.

de Graauw, T., Roelfsema, P., Helmich, F. et al. [2010] Astron. Astrophys. 518(2), 1.

Granet, C., James, G. L., Bolton, R. et al. [2004] IEEE Trans. Antennas Propag. 52(3), 848.
Heyminck, S. \& Graf, U. U. [2001] Proc. 12th Int. Symp. Space Terahertz Technol, Feb. 14-16, 2001, San Diego, CA, USA, p. 563.

Heyminck, S., Graf, U. U., Güsten, R. et al. [2012] Astron. Astrophys. 542, L1.

Justen, M., Bonzon, C., Ohtani, K. et al. [2016] Opt. Lett. 41(19), 4590.

Kasemann, C., Heyminck, S., Bell, A. et al. [2008] Proc. 19th Int. Symp. Space Terahertz Technol.

Kester, D., Avruch, I. \& Teyssier, D. [2014] AIP Conf. Proc. 1636(1), 62 .

Pabst, C. et al. [2018] submitted to Nature.

Pilbratt, G. et al. [2010] Astron. Astrophys. 518, L1.

Pütz, P., Honingh, C. E., Jacobs, K. et al. [2012] Astron. Astrophys. 542, L2.

Richter, H., Greiner-Bär, M., Pavlov, S. G. et al. [2010] Opt. Express 18, 10177.

Richter, H., Wienold, M., Schrottke, L. et al. [2015] IEEE Trans. Terahertz Sci. Technol. 5(4), 539.

Risacher C., Güsten, R., Stutzki, J. et al. [2016a] IEEE Trans. Terahertz Sci. Technol. 6(2), 199.

Risacher C., Güsten, R., Stutzki, J. et al. [2016b] Astron. Astrophys. 595A, 34R.

Schrottke, L., Wienold, M., Sharma R. et al. [2013] Semicond. Sci. Technol. 28, 035011.

Wagner-Gentner, A., Graf, U. U., Rabanus et al. [2006] Infrared Phys. Technol. 48, 249.

Walker, C. et al., "The Stratospheric $\mathrm{THz}$ Observatory (STO)", Proc. SPIE 7733, 77330N.

Young, E. T., Becklin, E. E., Marcum, P. M. et al. [2012] Astron. Astrophys. 749, L17. 